\title{
Lattice Theory for Finite Dimensional Hilbert Space with Variables in $\mathcal{Z}_{\mathbf{d}}$
}

\author{
Semiu Oladipupo Oladejo', Adediran Dauda Adeshola², Adedayo David Adeniyi ${ }^{1}$ \\ ${ }^{1}$ Department of Mathematics, Faculty of Science, Gombe State University, Gombe, Nigeria \\ ${ }^{2}$ Department of Statistical and Mathematical Science, College of Pure and Applied Science, Kwara State University, Ilorin, Nigeria \\ Email: abdsemiu@yahoo.com
}

How to cite this paper: Oladejo, S.O., Adeshola, A.D. and Adeniyi, A.D. (2019) Lattice Theory for Finite Dimensional Hilbert Space with Variables in $\mathcal{Z}_{\mathrm{d}}$. Journal of Quantum Information Science, 9, 111-121.

https://doi.org/10.4236/jqis.2019.92006

Received: December 18, 2019

Accepted: April 15, 2019

Published: April 18, 2019

Copyright $\odot 2019$ by author(s) and Scientific Research Publishing Inc. This work is licensed under the Creative Commons Attribution International License (CC BY 4.0).

http://creativecommons.org/licenses/by/4.0/

\begin{abstract}
In this work, join and meet algebraic structure which exists in non-near-linear finite geometry are discussed. Lines in non-near-linear finite geometry $\mathcal{Z}_{\mathbf{d}}^{2}$ were expressed as products of lines in near-linear finite geometry $\mathcal{Z}_{\mathfrak{p}}^{2}$ (where $\mathfrak{p}$ is a prime). An existence of lattice between any pair of near-linear finite geometry $\mathcal{Z}_{\mathfrak{p}_{\mathfrak{j}}}^{2},(\mathfrak{j}=1, \cdots, \mathfrak{k})$ of $\mathcal{Z}_{\mathbf{d}}^{2}$ is confirmed. For $\mathfrak{q} \mid \mathbf{d}$, a one-to-one correspondence between the set of subgeometry $\mathcal{Z}_{\mathfrak{q}_{j}}^{2}$ of $\mathcal{Z}_{\mathbf{d}}^{2}$ and finite geometry $\mathcal{Z}_{\mathbf{d}}^{2}$ from the subsets of the set $\{\mathcal{D}(\mathbf{d})\}$ of divisors of $\mathbf{d}$ (where each divisor represents a finite geometry) and set of subsystems $\{\Pi(\mathfrak{q})\}$ (with variables in $\mathcal{Z}_{\mathfrak{q}}$ ) of a finite quantum system $\Pi(\mathbf{d})$ with variables in $\mathcal{Z}_{\mathbf{d}}$ and a finite system from the subsets of the set of divisors of $\mathbf{d}$ is established.
\end{abstract}

\section{Keywords}

Lattice, Join, Meet, Least Upper Bound (LUB), Greatest Lower Bound (GLB), Partially Ordered Set (POSET)

\section{Introduction}

For quite some time, finite quantum systems with variables in $\mathcal{Z}_{\mathbf{d}}$ had received enormous attention [1] [2] [3] with special focus on mutually unbiased bases [4]-[9]. Likewise in recent times, the weak mutually unbiased base ( $\mathfrak{W M U} \mathfrak{B})$ is getting more interest from researchers [10] [11]. This might be due to the fact that they are concepts that have a significant role in quantum computation and information [12] [13] [14] [15]. Previously, most work done on finite geometry is on near-linear geometry. In this type of geometry two lines have at most one 
point in common [13]-[21]. In this work, we focus our attention on the structure of lattice found in lines in non-near-linear finite geometry and Hilbert space of finite quantum systems. A unique feature of our findings is that, any pair of small size finite geometry $\mathcal{Z}_{\mathrm{q}_{\mathrm{i}}}, \mathfrak{j}=1, \cdots, \mathfrak{k}$ of dimensions has a least upper bound (that is the meet) and greatest lower bound (the join). Furthermore, for any two prime dimesional finite geometry $\mathcal{Z}_{\mathfrak{p}_{\mathfrak{j}}}^{2}$ there is a reducible join and an irreducible meet. We partition this work into the following sections; the definitions and meaning of notations used in our work was discussed in Section 2 titled preliminaries. Section 3 covers the discussion of the non-near-linear geometry and its subgometries. In Section 4, we discuss the decomposition of lines in non-near-linear finite geometry $\mathcal{G}_{\mathrm{d}}$ and its subgometries $\mathcal{G}_{\mathfrak{q}}$ in relation to group lattice and sublattices. Group lattice and sublattices in the set of finite quantum system is discussed in Section 5. Finally in Section 6, we conclude our work.

\section{Preliminaries}

1) A POSET means a partially ordered set.

2) $\mathcal{Z}_{\mathbf{d}}$ represents ring of integer modulo $\mathbf{d}$.

3) $\left|\mathcal{Z}_{\mathbf{d}}^{*}\right|$ is $\varphi(\mathbf{d})$ where $\mathcal{Z}_{\mathbf{d}}^{*}$ represents the set of invertible elements in $\mathcal{Z}_{\mathbf{d}}$ and $\varphi(\mathbf{d})$ is referred to as Euler Phi function. It is defined as

$$
\varphi(\mathbf{d})=\mathbf{d} \prod_{j=1}^{\mathfrak{k}}\left(1-\frac{1}{\mathfrak{p}_{j}}\right)
$$

4) $\psi(\mathbf{d})$ is called the Dedekind psi function where;

$$
\psi(\mathbf{d})=\mathbf{d} \prod_{j=1}^{\mathfrak{k}}\left(1+\frac{1}{\mathfrak{p}_{j}}\right) ; \mathfrak{p}_{j}=\text { prime }
$$

5) The notation $\{\mathcal{D}(\mathbf{d})\}$ represents the set of proper divisors of $\mathbf{d}$ and any pairs of divisors form a lattice in our work. It is a POSET with divisibility as partial order. The number of element in this set is the divisor function $\sigma_{0}(\mathbf{d})$. where

$$
\sigma_{0}(\mathbf{d})=\sum_{\mathfrak{q} \mid \mathbf{d}} \mathfrak{q}^{0}
$$

It forms a complete lattice in $\mathcal{G}_{\mathrm{d}}$.

6) In this paper, we use the symbol $\prec$ to represent partial order. If $q$ is a factor of $\mathbf{d}$ then $\mathfrak{q} \prec \mathbf{d}$ and $\mathcal{Z}_{\mathfrak{q}}$ is a subgroup of $\mathcal{Z}_{\mathbf{d}}$.

7) We define the set of subgroup of $\mathcal{Z}_{\mathbf{d}}$ as

$$
\mathbb{Z}_{\mathbf{d}}=\left\{\mathcal{Z}_{\mathfrak{q}} \mid \mathfrak{q} \in \mathcal{D}(\mathbf{d})\right\}
$$

It is a partially ordered set with divisibility as partial order. There is a bijection between the set $\{\mathcal{D}(\mathbf{d})\}$ of divisors of $\mathbf{d}$ and $\mathcal{Z}_{\mathfrak{q}}$ where $\mathfrak{q}$ represents a divisor of $\mathbf{d}$. The elements of $\mathcal{Z}_{\mathfrak{q}}$ are embedded in $\mathcal{Z}_{\mathbf{d}}$ for $\mathfrak{q} \mid \mathbf{d}$ thus

$$
\alpha \in \mathcal{Z}_{\mathfrak{q}} \rightarrow \frac{\mathbf{d} \alpha}{\mathfrak{q}} \in \mathcal{Z}_{\mathbf{d}} .
$$


8) The notations: $\wedge$ and $\vee$ denote meet or roof and floor or join respectively. The greatest common divisor of two element $\alpha$ and $\beta$ is represented in this work as $\mathcal{G C D}(\alpha, \beta)$.

9) We express d as

$$
\mathbf{d}=\mathfrak{p}_{1} \times \mathfrak{p}_{2} \times \cdots \times \mathfrak{p}_{\mathfrak{k}} .
$$

Mathematically, $\mathcal{Z}_{\mathbf{d}}$ is a cyclic module.

This work focuses on non-near-linear geometry. That is, in this case two lines for example intersects in more than one points. It is related to the fact that $\mathcal{Z}_{\mathbf{d}}$ is a ring of integer modulo $\mathbf{d}$ and all the lines in this work are through the origin.

\section{Non-Near-Linear Geometry $\mathcal{G}_{\mathrm{d}}$ and Its Subgometries $\mathcal{G}_{\mathfrak{q}}$}

We define the finite geometry $\mathcal{G}_{\mathbf{d}}=\mathcal{Z}_{\mathbf{d}}^{2}$ as the combination

$$
\mathcal{G}_{\mathrm{d}}=\left(\mathcal{P}_{\mathrm{d}}, \mathcal{L}_{\mathrm{d}}\right) \text {. }
$$

$\mathcal{P}_{\mathbf{d}}$ represents points on a line and $\mathcal{L}_{\mathrm{d}}$ represent lines in $\mathcal{G}_{\mathrm{d}}$ where

$$
\mathcal{P}_{\mathbf{d}}=\left\{(e, f) \mid e, f \in \mathcal{Z}_{\mathbf{d}}\right\} .
$$

Definition 3.1. A line through the origin is defined as

$$
\mathfrak{L}(\alpha, \beta)=\left\{(s \alpha, s \beta) \mid \alpha, \beta \in \mathcal{Z}_{\mathrm{d}}\right\} s \in \mathcal{Z}_{\mathrm{d}}
$$

In this work $\prod_{\mathrm{j}=1}^{\mathfrak{k}} \mathcal{G}_{\mathfrak{p}_{\mathrm{j}}}$ and $\prod_{\mathrm{j}=1}^{\mathrm{k}} \mathcal{Z}_{\mathrm{p}_{\mathrm{j}}}^{2}$ represents the same concept, so as a result we use them interchangeably.

Definition 3.2. We define a lattice as a POSET in which every pair of elements have the LUB (or the join, $\vee$ ) and GLB (or the meet, $\wedge$ ). In this work each element of the set $\{\mathcal{D}(\mathbf{d})\}$ represents a finite geometry.

From the results of [10] [11] [12] we confirm the following propositions without proof:

Proposition 3.3. 1) If

$$
b \in \mathcal{Z}_{\mathbf{d}}^{*} \text { then } \mathfrak{L}(\alpha, \beta)=\mathfrak{L}(b \alpha, b \beta) .
$$

also, if

$$
\begin{aligned}
b \in \mathcal{Z}_{\mathbf{d}}-\mathcal{Z}_{\mathbf{d}}^{*} \text { then } \mathfrak{L}(\alpha, \beta) \bmod (\mathbf{d}) \subset \mathfrak{L}(b \alpha, b \beta), \\
\text { hence } \mathfrak{L}(b \alpha, b \beta) \prec \mathfrak{L}(\alpha, \beta)
\end{aligned}
$$

We confirmed that $\mathfrak{L}(\alpha, \beta)$ is a maximal line in $\mathcal{G}_{\mathbf{d}}$ if $\mathcal{G C D}(\alpha, \beta) \in \mathcal{Z}_{\mathbf{d}}^{*}$ and $\mathfrak{L}(\alpha, \beta)$ is a subline in $\mathcal{G}_{\mathbf{d}}$ if $\mathcal{G C D}(\alpha, \beta) \in \mathcal{Z}_{\mathbf{d}}-\mathcal{Z}_{\mathbf{d}}^{*}$.

2) The number of maximal lines in $\mathcal{G}_{\mathbf{d}}$ with $\mathbf{d}$ points each is $\psi(\mathbf{d})$.

3) Suppose we define a line in finite geometry $\mathcal{G}_{\mathbf{d}}$ as in Equation (9), $\mathfrak{L}(\alpha, \beta)$ is also

$$
\mathfrak{L}(s \alpha, s \beta)=\left\{(s \xi \alpha, s \xi \beta) \mid \xi \in \mathcal{Z}_{\mathrm{d}}\right\} \text {, in } \mathcal{G}_{\xi \mathrm{d}}
$$

at the same time the line $\mathfrak{L}(\xi \alpha, \xi \beta)$ in $\mathcal{G}_{\xi \mathrm{d}}$ is a subline of

$$
\mathfrak{L}(\alpha, \beta)=\left\{\left(s^{\prime} \alpha, s^{\prime} \beta\right) \mid s^{\prime}=0, \cdots, \xi \mathbf{d}-1\right\},
$$


4) If two maximal lines have $\mathfrak{q}$ points in common where $\mathfrak{q} \mid \mathbf{d}$. The $\mathfrak{q}$ points gives a subline $\mathfrak{L}(\alpha, \beta)$ where $\alpha, \beta \in \frac{\mathbf{d}}{\mathfrak{q}} \mathcal{Z}_{\mathfrak{q}}$.

If we consider the subgeometry $\mathcal{G}_{\mathfrak{q}}$, the subline $\mathfrak{L}(\alpha, \beta)$ in $\mathcal{G}_{\mathbf{d}}$ is a maximal line in $\mathcal{G}_{\mathfrak{q}}$. There is $\psi(\mathfrak{q})$ maximal lines in subgeometry $\mathcal{G}_{\mathfrak{q}}$ of finite geometry $\mathcal{G}_{\mathbf{d}}$.

\section{Factorization of Lines in Non-Near-Linear Geometry as Lines in Near-Linear Geometries}

In this section, each lines in $\mathcal{Z}_{\mathbf{d}}^{2}$ is decomposed as lines in $\prod_{\mathrm{j}=1}^{\mathfrak{k}} \mathcal{Z}_{\mathfrak{p}_{\mathrm{j}}}^{2}$. Using the concept of Good [17] two bijective maps were created between the ordinates of each of the lines in non-near-linear geometry. Similar concept was used in the past by [1] [10] [11] to factorize a large finite dimensional finite quantum systems as products of many small dimensional finite systems that is,

$$
\begin{gathered}
\gamma \leftrightarrow\left(\gamma_{1}, \cdots, \gamma_{\mathfrak{k}}\right) ; \gamma_{j}=\gamma\left(\bmod \mathfrak{p}_{j}\right) ; \gamma=\sum \gamma_{j} \mathbf{s}_{j} \\
\gamma \leftrightarrow\left(\bar{\gamma}_{1}, \cdots, \bar{\gamma}_{\mathfrak{k}}\right) ; \bar{\gamma}_{j}=\gamma \mathbf{t}_{j}=\gamma_{j} \mathbf{t}_{j}\left(\bmod \mathfrak{p}_{j}\right) ; \gamma=\sum \bar{\gamma}_{j} \mathbf{r}_{\mathfrak{j}}(\bmod \mathbf{d})
\end{gathered}
$$

where

$$
\mathbf{r}_{j}=\frac{\mathbf{d}}{\mathfrak{p}_{j}} ; \mathbf{t}_{j} \mathbf{r}_{\mathfrak{j}}=1\left(\bmod \mathfrak{p}_{\mathrm{j}}\right) ; \mathbf{s}_{\mathrm{j}}=\mathbf{t}_{\mathrm{j}} \mathbf{r}_{\mathrm{j}} \in \mathcal{Z}_{\mathbf{d}} .
$$

Equation (15) and Equation (16) represent position and momentum states respectively. We used the above bijection in our earlier work [12] to factorize maximal lines in $\mathcal{G}_{\mathbf{d}}$ as prime factor lines $\mathcal{G}_{\mathfrak{p}_{\mathfrak{j}}}$. There is a bijection between the set of lines of $\mathcal{G}_{\mathbf{d}}$ that is,

$$
\mathfrak{L}(x, y) \text { in } \mathcal{Z}_{\mathbf{d}}^{2}
$$

and prime factor lines $\mathcal{G}_{\mathfrak{p}_{j}}$ that is

$$
\mathfrak{L}\left(x_{1}, \bar{y}_{1}\right) \times \cdots \times \mathfrak{L}\left(x_{\mathfrak{k}}, \bar{y}_{\mathfrak{k}}\right) \text { in } \prod_{\mathfrak{j}=1}^{\mathfrak{k}} \mathcal{Z}_{\mathfrak{p}_{\mathfrak{j}}}^{2}
$$

where

$$
(x, y) \leftrightarrow\left(x_{1}, \bar{y}_{1}\right) \times \cdots \times\left(x_{\mathfrak{k}}, \bar{y}_{\mathfrak{k}}\right) \text { and } \mathfrak{p}_{j} \text { a prime }
$$

We confirmed the existence of $\mathbf{d}^{2}-1$ maximal lines altogether. Out of which there are only $\psi(\mathbf{d})$ maximal lines are distinct. We also confirm that each distinct lines has $\varphi(\mathbf{d})$ equivalent lines whose all its points map the points of each of the distinct lines in the non-near-linear finite geometry $\mathcal{G}_{\mathbf{d}}$ and as a result we confirm the existence of an equivalence relation between all the points in each of the maximal lines and other $\varphi(\mathbf{d})$ lines in the non-near-linear geometry. Also we discovered that each of the factored lines in $\mathcal{G}_{\mathfrak{p}_{\mathfrak{j}}}$ is a maximal lines in $\mathcal{G}_{\mathfrak{q}}$ and at the same time a subline in $\mathcal{G}_{\mathbf{d}}$. In addition, if we take any two arbitrary maximal lines one from each near-linear geometry $\mathcal{G}_{\mathfrak{p}_{\mathfrak{j}}}, \mathfrak{j}=1, \cdots, \mathfrak{k}$, the two lines join to form a subline $\mathcal{G}_{\mathfrak{q}}$ of $\mathcal{G}_{\mathbf{d}}$ and at the same time taking the intersection of the near-linear geometries gives a meet which is a subgeometry of the two prime 
geometries. Hence $\mathcal{G}_{\mathfrak{p}_{1}}$ and $\mathcal{G}_{\mathbf{p}_{2}}$ form a lattice of $\mathcal{G}_{\mathrm{d}}$.

In this work, the term decomposition is analogous to factorization of non-prime integers as products of their primes. The geometry $\mathcal{G}_{\mathbf{d}}$ is related to the set of divisors $\mathcal{D}(\mathbf{d})$ of $\mathbf{d}$, the subline $\mathfrak{L}(s X, s y)$ in $\frac{\mathbf{d}}{\mathfrak{q}} \mathcal{Z}_{\mathfrak{q}} \times \frac{\mathbf{d}}{\mathfrak{q}} \mathcal{Z}_{\mathfrak{q}}$ is related to common divisor between two or more integers and $\mathfrak{L}(0,0)$ corresponds to a line which contains only one point $(0,0)$.

The factorization is related to finding the lowest common multiple (L.C.M.) of a set of integer, the L.C.M. represents the roof. The H.C.F. of any two prime geometries is connected to finding the intersection any two disjoint sets. In this work we call the H.C.F. the floor. As an illustration, we define line in $\mathcal{Z}_{\mathbf{d}}^{2}$ as in Equation (19) thus.

Suppose $x, y \in \mathcal{Z}_{\mathbf{d}}$ for $\mathbf{d}$ a non-prime, not every element in $\mathcal{Z}_{\mathbf{d}}$ has a multiplicative inverse and so as a result Equation (19) is expressed further as,

$$
\mathfrak{L}(x, y)=\mathfrak{L}\left(1, x_{1}^{-1} \bar{y}_{1}\right) \times \cdots \times \mathfrak{L}\left(1, x_{\mathfrak{k}}^{-1} \bar{y}_{\mathfrak{k}}\right)
$$

here we represent

$$
\Theta_{\mathfrak{k}} \equiv x_{\mathfrak{k}}^{-1} y_{\mathfrak{k}}
$$

therefore $x \neq 0$;

$$
\mathfrak{L}(x, y)=\Gamma\left(\Theta_{1}\right) \times \cdots \times \Gamma\left(\Theta_{\mathfrak{k}}\right)=\Gamma\left(\Theta_{1} \cdots \Theta_{\mathfrak{k}}\right) .
$$

However, for $x_{\mathfrak{l}}=0$, the line $\mathfrak{L}\left(0, y_{\mathfrak{k}}\right)=\mathfrak{L}(0,1)=\mathfrak{L}(1,-1) \equiv \Gamma(-1)$.

As an illustration, we express all maximal lines in $\mathcal{G}_{\mathbf{d}}=\mathcal{Z}_{\mathbf{d}}^{2}$ for $\mathbf{d}=6$ in terms of its primes discussed in Equation (21) and Equation (23) above by decomposing line $\mathfrak{L}(2,1)$.

Using Equation (15) the ordinate 2 in $\mathfrak{L}(1,2)$ is decomposed as;

$$
2 \leftrightarrow(0,2)
$$

also using Equation (16) the ordinate 1 in $\mathfrak{L}(1,2)$ is decomposed as;

$$
1 \leftrightarrow(1,2)
$$

Therefore $\mathfrak{L}(2,1)$ is decomposed as;

$$
\mathfrak{L}(0,1) \times \mathfrak{L}(2,2) .
$$

if we relate Equation (26) to Equation (21) and Equation (23), $\mathfrak{L}(2,1)$ is expressed as

$$
\mathfrak{L}(1,-1) \times \mathfrak{L}(1,1) \equiv \Gamma(-1,1) .
$$

Here we use Equation (15) and Equation (16) to express $\psi(6)=12$ maximal lines in $\mathcal{G}_{6}$ and its subgometries $3 \mathcal{G}_{2}$ and $2 \mathcal{G}_{3}$ as partition in Table 1 where $\mathcal{G}_{6}$ is isomorphic to $\mathcal{G}_{2}$ and $\mathcal{G}_{3}$. Suppose $\mathbf{d}=6, \mathfrak{p}_{1}=2, \mathfrak{p}_{2}=3, \mathfrak{t}_{1}=1$, $\mathbf{t}_{2}=2, \mathbf{r}_{1}=3$ and $\mathbf{r}_{2}=2$

Proposition 4.1. 1) Suppose $\mathcal{G}_{\mathbf{d}}=\prod_{\mathrm{j}=1}^{\mathfrak{k}} \mathcal{G}_{\mathfrak{p}_{\mathrm{j}}}$ is a non-near-linear finite geometry, then the set of near-linear geometries $\mathcal{G}_{\mathfrak{p}_{\mathfrak{j}}}$ (for $\mathfrak{p}$ a prime) obtained through factorizing the non-near-linear geometry forms a lattice, and as a result forms a partition. 
Table 1. Maximal lines in non-near-linear finite geometries G6 in terms of its prime factor lines.

\begin{tabular}{ccccc}
\hline $\mathcal{G}_{6} \cong \mathcal{G}_{2} \oplus \mathcal{G}_{3}$ & $\mathbb{A}_{1}$ & $\mathbb{A}_{2}$ & $\mathbb{A}_{3}$ & $2 \mathcal{G}_{3}=\mathbb{A}_{1} \cap \mathbb{A}_{2} \cap \mathbb{A}_{3}$ \\
\hline $\mathbb{B}_{1}$ & $\mathfrak{L}(0,1)=\Gamma(-1,-1)$ & $\mathfrak{L}(3,2)=\Gamma(0,-1)$ & $\mathfrak{L}(3,1)=\Gamma(1,-1)$ & $\mathfrak{L}(0,2)=\Gamma(-1)$ \\
$\mathbb{B}_{2}$ & $\mathfrak{L}(2,3)=\Gamma(-1,0)$ & $\mathfrak{L}(1,0)=\Gamma(0,0)$ & $\mathfrak{L}(1,3)=\Gamma(1,0)$ & $\mathfrak{L}(2,0)=\Gamma(0)$ \\
$\mathbb{B}_{3}$ & $\mathfrak{L}(2,5)=\Gamma(-1,2)$ & $\mathfrak{L}(1,4)=\Gamma(0,2)$, & $\mathfrak{L}(1,1)=\Gamma(1,2)$ & $\mathfrak{L}(2,2)=\Gamma(1)$ \\
$\mathbb{B}_{4}$ & $\mathfrak{L}(2,1)=\Gamma(-1,1)$ & $\mathfrak{L}(1,2)=\Gamma(0,1)$ & $\mathfrak{L}(1,5)=\Gamma(1,1)$ & $\mathfrak{L}(2,4)=\Gamma(2)$ \\
$3 \mathcal{G}_{2}=\mathbb{B}_{1} \cap \mathbb{B}_{2} \cap \mathbb{B}_{3} \cap \mathbb{B}_{4}$ & $\mathfrak{L}(0,3)=\Gamma(-1)$ & $\mathfrak{L}(3,0)=\Gamma(0)$ & $\mathfrak{L}(3,3)=\Gamma(1)$ & \\
\hline
\end{tabular}

Proof: Since $\mathcal{G}_{\mathfrak{p}_{\mathfrak{j}}}, \mathfrak{j}=1,2, \cdots, \mathfrak{k}$ are near linear geometries and taking the intersection of any two lines $\mathfrak{L}_{1}(x, y)$ and $\mathfrak{L}_{2}(a, b) \in \mathcal{G}_{\mathfrak{p}_{1}}, a, b, x, y, \in \mathcal{Z}_{\mathfrak{p}_{1}}$ yields only line $\mathfrak{L}(0,0)$ which is the trivial near-linear geometry $\mathcal{G}_{1}$. Hence the proof is complete.

2) Two lines in $\mathcal{G}_{d}$ are isomorphic if there is a 1-1 correspondence between the points in $\mathfrak{L}_{1}$ and $\mathfrak{L}_{2}$.

Proof: Since $\mathfrak{L}_{1}(x, y)$ and $\mathfrak{L}_{2}(r x, r y) \in \mathcal{G}_{\mathbf{d}}, r \in \mathcal{Z}_{\mathbf{d}}^{*}$ and the existence of bijection between the points in $\mathfrak{L}_{1}(x, y)$ and $\mathfrak{L}_{2}(x, y)$ make itself evident.

\subsection{Symplectic Group on $\mathcal{G}_{\mathrm{d}}$}

We define the matrices

$$
\mathcal{M}(\delta, \eta \mid \lambda, \theta)
$$

where $\mathcal{M}(\delta, \eta \mid \lambda, \theta) \equiv\left(\begin{array}{ll}\delta & \eta \\ \lambda & \theta\end{array}\right)$

$$
\operatorname{det} \mathcal{M}=(\delta \theta-\eta \lambda)=1(\bmod \mathbf{d}) ; \text { where } \delta, \eta, \lambda, \theta \in \mathcal{Z}_{\mathbf{d}}
$$

$\mathcal{M}$ form a group called symplectic group $\operatorname{Sp}\left(2, \mathcal{Z}_{\mathbf{d}}\right)$ group.

Suppose we act $\mathcal{M}$ on all points of line $\mathfrak{L}(x, y)$ in $\mathcal{Z}_{\mathbf{d}}^{2}$. This produces all the points of the line $\mathfrak{L}(\delta x+\eta y, \lambda x+\theta y)$. We write it as $\mathcal{M}(\delta, \eta \mid \lambda, \theta) \mathfrak{L}(x, y)$. Suppose d is a prime, acting $\mathcal{M}(0,1 \mid-1, \Theta)$ on the line $\mathfrak{L}(0,1)$, we obtain all the lines (maximal lines) through the origin. In this work, we label the lines as

$$
\begin{gathered}
\Theta=-1 \rightarrow \Gamma(-1)=\mathfrak{L}(0,1), \\
\Theta=0, \cdots,-1 \rightarrow \Gamma(\Theta)=\mathcal{M}(0,1 \mid-1, \Theta) \mathfrak{L}(0,1)=\mathfrak{L}(1, \Theta) .
\end{gathered}
$$

In this work, we take the condition that for $\Theta=-1, \mathcal{M}(0,1 \mid-1, \Theta)$ is replaced by $\mathcal{M}(1,0 \mid 0,1)$.

Thus, $\operatorname{Sp}\left(2, \mathcal{Z}_{\mathbf{d}}\right)$ is expressed as $\operatorname{Sp}\left(2, \mathcal{Z}_{\mathrm{p}_{1}}\right) \times \cdots \times \operatorname{Sp}\left(2, \mathcal{Z}_{\mathfrak{p}_{\mathfrak{k}}}\right)$

$$
\mathcal{M}(\delta, \eta \mid \lambda, \theta)=\otimes_{\mathrm{i}} \mathcal{M}\left(\delta_{\mathrm{j}}, \mathbf{r}_{\mathrm{j}} \eta_{\mathrm{j}} \mid \bar{\lambda}_{\mathrm{j}}, \theta_{\mathrm{j}}\right),
$$

where $\delta_{\mathrm{j}}, \eta_{\mathrm{j}}, \theta_{\mathrm{j}}$ are related $\delta, \eta, \theta$ in Equation (15) and $\lambda_{\mathrm{j}}$ is related to $\lambda$ in Equation (16).

Any pair of geometry in the set form a lattice and the set $\left\{\mathbb{G}_{\mathbf{d}}\right\}$ of all subgometries of $\mathcal{G}_{\mathbf{d}}$ is isomorphic to the set $\{\mathcal{D}(\mathbf{d})\}$. 


\subsection{Join Reducible and Meet Irreducible in Finite Geometry}

In this subsection, we discuss how the union of two or more near-linear geometries $\mathcal{G}_{\mathbf{p}_{\mathbf{i}}}$ forms subgometries $\mathcal{G}_{\mathfrak{q}}$ of non-near-linear geometry and their intersection produces maximal lines in near-linear geometry $\mathcal{G}_{\mathfrak{p}}$ via partial ordering and as a result forms a lattice. Suppose we define the set of geometry

$$
\begin{aligned}
& \mathcal{I}=\{1, \cdots, N\} ; \text { where } \mathcal{I}_{1} \subset \mathcal{I}, \\
& \mathcal{I}_{1} \vee \mathcal{I}_{2}=\operatorname{LUB}\left(\mathcal{I}_{1}, \mathcal{I}_{2}\right)=\text { Join }
\end{aligned}
$$

and

$$
\mathcal{I}_{1} \wedge \mathcal{I}_{2}=G L B\left(\mathcal{I}_{1}, \mathcal{I}_{2}\right)=\text { Meet }
$$

\subsection{Examples}

Suppose $\mathbf{d}=42, \mathbb{G}_{42}=\left\{\left\{\mathcal{G}_{42}\right\},\left\{\mathcal{G}_{21}\right\},\left\{\mathcal{G}_{14}\right\},\left\{\mathcal{G}_{6}\right\},\left\{\mathcal{G}_{7}\right\},\left\{\mathcal{G}_{3}\right\},\left\{\mathcal{G}_{2}\right\},\left\{\mathcal{G}_{1}\right\}\right\}$.

If we take the set $\left\{\mathcal{G}_{21}\right\}$ and $\left\{\mathcal{G}_{14}\right\}:\left\{\left\{\mathcal{G}_{21}\right\} \vee\left\{\mathcal{G}_{14}\right\}\right\}=\left\{\mathcal{G}_{42}\right\}$, and $\left\{\mathcal{G}_{21}\right\} \wedge\left\{\mathcal{G}_{14}\right\}=\left\{\mathcal{G}_{7}\right\}$.

That is the maximal lines in $\mathcal{G}_{21}$ and $\mathcal{G}_{14}$ are sublines in $\mathcal{G}_{42}$ and the intersection of sublines is isomorphic to maximal lines in $\mathcal{G}_{7}$. In this case $\mathcal{G}_{42}$, they form a join in $\mathcal{G}_{42}$ and a meet in $\mathcal{G}_{7} . \mathcal{G}_{42}$ and $\mathcal{G}_{7}$ are called the $L U B$ and $G L B\left\{\mathcal{G}_{21}\right\}$ and $\left\{\mathcal{G}_{14}\right\}$ respectively. This forms a complete lattice in $\left\{\mathcal{G}_{42}\right\}$. Likewise the three sets, $\left\{\mathcal{G}_{14}\right\},\left\{\mathcal{G}_{21}\right\}$, and $\left\{\mathcal{G}_{42}\right\}$ are sublattices in $\left\{\mathcal{G}_{210}\right\}$. The subgometries $7 \mathcal{G}_{6}$ and $6 \mathcal{G}_{7}$ is isomorphic to $\mathcal{G}_{6}$ and $\mathcal{G}_{7}$ respectively.

The join is analogous to non prime integers which can be expressed as products of prime integers, while the meet is related to the factors of such non prime integers which when one factorizes further it get to a point where there the only factor it will have is the integer 1.

More examples are shown in Table 2 and Hasse diagram (Figure 1).

Table 2. A table of maximal lines in non-near-linear finite geometry $\mathcal{G}_{42}$ and its subgometries.

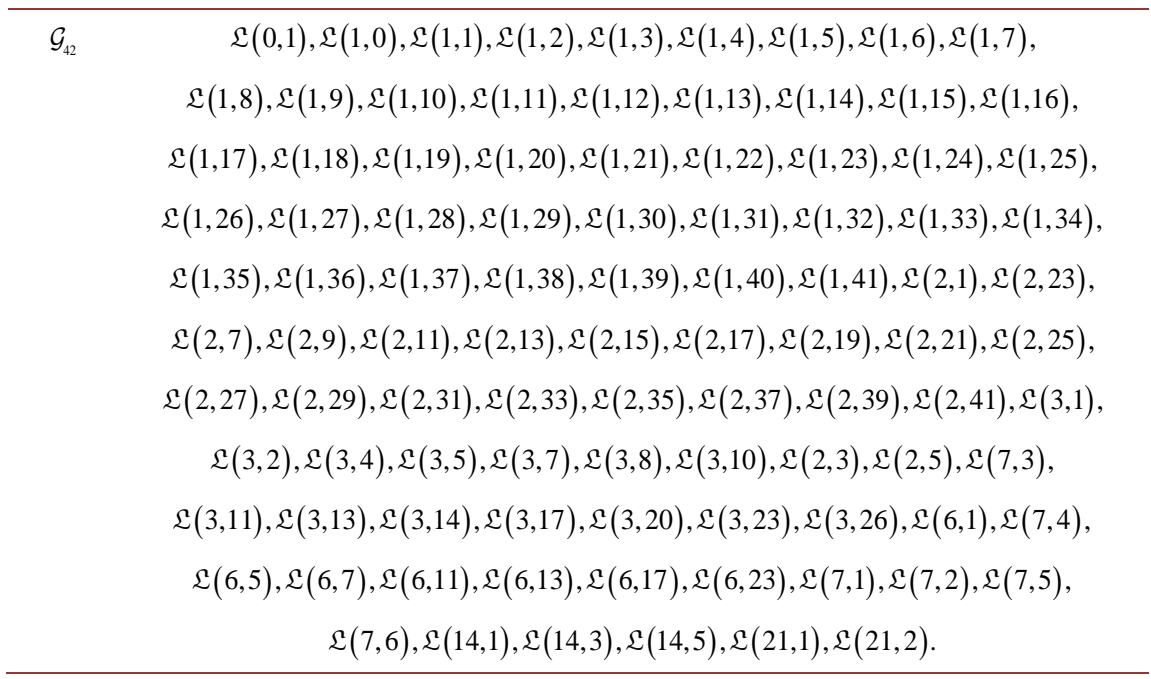




\section{Continued}
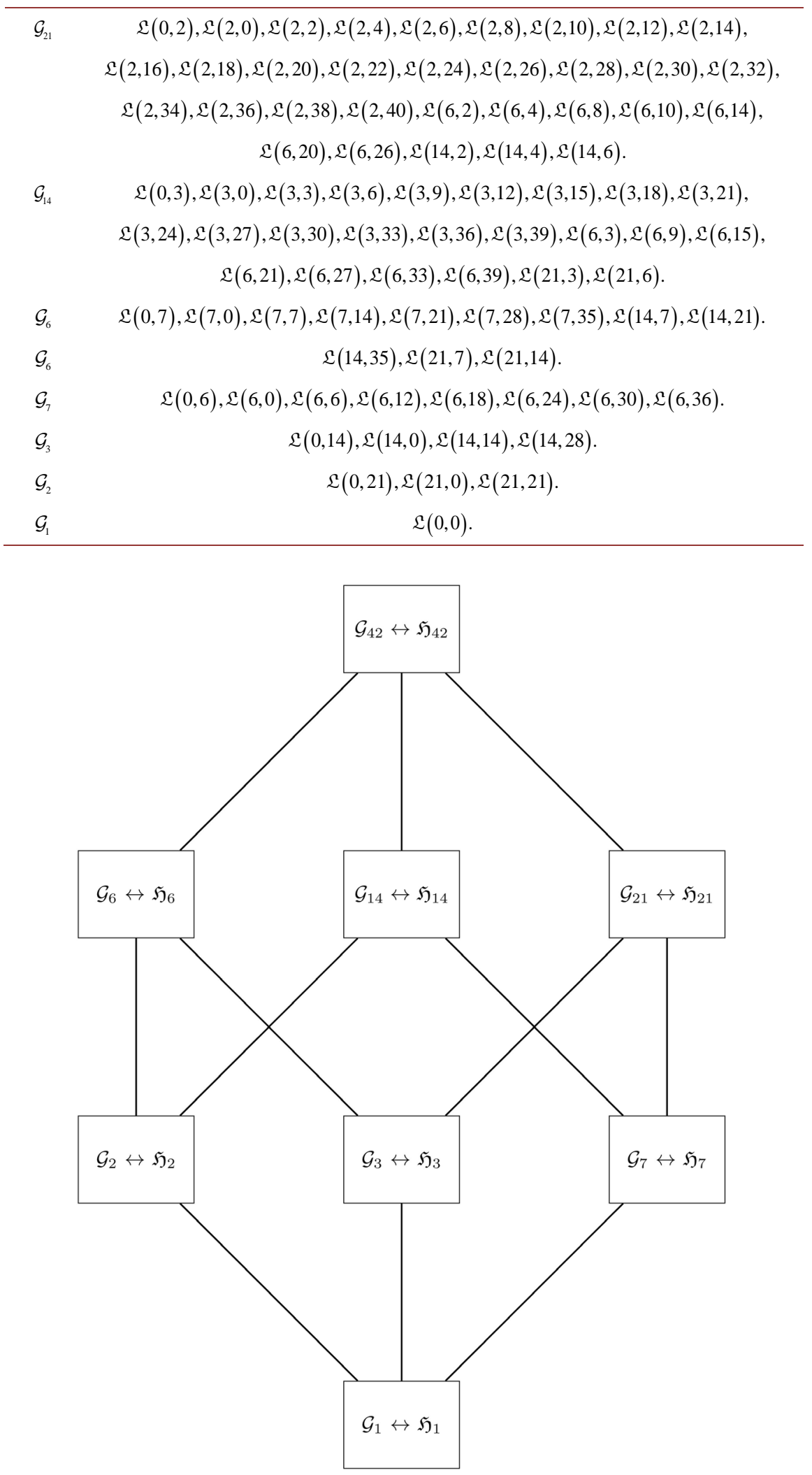

Figure 1. The Hasse diagram showing the non-near-linear geometry $\mathcal{G}_{42}$ and its subgometries, and along with Hilbert spaces $\mathfrak{H}_{42}$ of the subsystems of $\Pi(42)$. 


\section{Lattice Theory for Finite Dimensional Hilbert Space with Variables in $\mathcal{Z}_{\mathrm{d}}$}

We consider a quantum system with positions and momenta in $\mathcal{Z}_{\mathbf{d}}$, which we denote as $\Pi(\mathbf{d})$. For $\mathfrak{q}$ a divisor of $\mathbf{d}, \mathcal{Z}_{\mathfrak{q}}$ is a subgroup of $\mathcal{Z}_{\mathbf{d}}$. In this case we say that $\Pi(\mathfrak{q})$ is a subsystem of $\Pi(\mathbf{d})$.

Let $\left|\mathcal{X}_{\mathbf{d}} ; m\right\rangle$ and $\left|\mathcal{P}_{\mathbf{d}} ; m\right\rangle$ be position and momentum states, respectively. Here the $\mathcal{X}_{\mathbf{d}}, \mathcal{P}_{\mathbf{d}}$ are not variables but rather represent position and momentum respectively, in the $\mathbf{d}$-dimensional quantum system. The variables of $m$ belongs to $\mathcal{Z}_{\mathbf{d}}$. The Fourier transform is given by:

$$
\mathcal{F}_{\mathbf{d}}=\mathbf{d}^{-\frac{1}{2}} \sum_{m, \mathcal{N}=0}^{\mathbf{d}-1} \Omega(m \mathcal{N})\left|\mathcal{X}_{\mathbf{d}} ; m\right\rangle\left\langle\mathcal{X}_{\mathbf{d}} ; \mathcal{N}\right| ; \Omega(m)=\exp \left(i \frac{2 \pi m}{\mathbf{d}}\right)
$$

where $\left|\mathcal{P}_{\mathbf{d}} ; m\right\rangle=\mathcal{F}_{\mathbf{d}}\left|\mathcal{X}_{\mathbf{d}} ; m\right\rangle$ We define the displacement operator $D(v, \mu)$ as

$$
D(v, \mu)=Z^{v} X^{\mu} \Omega\left(-2^{-1} v \mu\right)
$$

where

$$
Z^{v}=\sum_{\mathcal{N}=0}^{\mathbf{d}-1} \Omega(\mathcal{N} v)\left|\mathcal{X}_{\mathbf{d}} ; \mathcal{N}\right\rangle\left\langle\mathcal{X}_{\mathbf{d}} ; \mathcal{N}\right|
$$

and

$$
X^{\mu}=\sum_{\mathcal{N}=0}^{\mathbf{d}-1} \Omega(-\mathcal{N} \mu)\left|\mathcal{P}_{\mathbf{d}} ; \mathcal{N}\right\rangle\left\langle\mathcal{P}_{\mathbf{d}} ; \mathcal{N}\right|
$$

where Equation (36) and Equation (37) satisfy the condition:

$$
X^{\mu} Z^{v}=Z^{v} X^{\mu} \Omega(-v \mu) ; X^{\mathbf{d}}=Z^{\mathbf{d}}=1
$$

The $D(v, \mu) \Omega(\lambda)$ where $v, \mu, \lambda \in \mathcal{Z}_{\mathbf{d}}$ form a representation of Heisenberg-Weyl group. References [2] [10] used Equation (15) and Equation (16) to decompose a system with variables in $\mathcal{Z}_{\mathbf{d}}$, where $\mathbf{d}$ is given in Equation (6), in terms of $\mathfrak{k}$ subsystems with variables in $\mathcal{Z}_{\mathfrak{p}_{\mathfrak{j}}}$. The existence of one-to-one correspondence between $\mathfrak{H}_{\mathbf{d}}$ and the tensor product $\otimes_{\mathrm{j}=1}^{\mathfrak{k}} \mathfrak{H}_{\mathfrak{p}_{\mathfrak{j}}}$ is confirmed where

$$
\left|\mathcal{X}_{\mathbf{d}} ; \gamma\right\rangle \leftrightarrow\left|\mathcal{X}_{\mathfrak{p}_{1}} ; \bar{\gamma}_{1}\right\rangle \otimes \cdots \otimes\left|\mathcal{X}_{\mathfrak{p}_{\mathfrak{k}}} ; \bar{\gamma}_{\mathfrak{k}}\right\rangle
$$

The same analogy is done for momentum basis thus;

$$
\left|\mathcal{P}_{\mathbf{d}} ; \gamma\right\rangle \leftrightarrow\left|\mathcal{P}_{\mathfrak{p}_{1}} ; \gamma_{1}\right\rangle \otimes \cdots \otimes\left|\mathcal{P}_{\mathfrak{p}_{\mathfrak{k}}} ; \gamma_{\mathfrak{k}}\right\rangle
$$

\section{Embedding of Small Systems into Large Systems}

If $\mathfrak{q} \mid \mathbf{d}$ then $\mathcal{Z}_{\mathfrak{q}} \subset \mathcal{Z}_{\mathbf{d}}$ also means that $\Pi(\mathfrak{q})$ is a subsystem of $\Pi(\mathbf{d})$.

In quantum states, $\Pi(\mathfrak{q})$ which takes variables in $\mathcal{Z}_{\mathfrak{q}}$ is embedded in $\Pi(\mathbf{d})$ which takes values in $\mathcal{Z}_{\mathbf{d}}$.

We express it as

$$
\sum_{m=0}^{\mathfrak{q}-1} \mathcal{S}_{m}\left|\mathcal{X}_{\mathfrak{q}} ; m\right\rangle \rightarrow \sum_{m=0}^{\mathfrak{q}-1} \mathcal{S}_{m}\left|\mathcal{X}_{\mathbf{d}} ; \frac{\mathbf{d} m}{\mathfrak{q}}\right\rangle
$$

The momentum representation is expressed as 


$$
\sum_{m=0}^{\mathfrak{q}-1} \mathcal{T}_{m}\left|\mathcal{P}_{\mathfrak{q}} ; m\right\rangle \rightarrow \sum_{m=0}^{\mathfrak{q}-1} \mathcal{T}_{m}\left|\mathcal{P}_{\mathbf{d}} ; \frac{\mathbf{d} m}{\mathfrak{q}}\right\rangle
$$

Hence the set $\{\Xi(\mathfrak{q})\}$ of subsystems, $\Pi(\mathfrak{q})$ of $\Pi(\mathbf{d})$ is isomorphic to the set $\{\mathcal{D}(d)\}$ and form a complete lattice in $\Pi(\mathbf{d})$.

\section{Conclusion}

Our central focus in this work is on the concept of lattice which exists in non-near-linear finite geometry $\mathcal{G}_{\mathrm{d}}$ and prime geometries $\mathcal{G}_{\mathfrak{p}_{\mathfrak{k}}}$ and the finite quantum system $\Pi(\mathbf{d})$ and its subsystem $\Pi(\mathfrak{q})$ with subsystems forming a lattice. More importantly, the complexity shown in this work demonstrates those important relations which exist between stucture and its substructures both in quantum system and geometry in its phase space.

\section{Conflicts of Interest}

The authors declare no conflicts of interest regarding the publication of this paper.

\section{References}

[1] Vourdas, A. (2004) Quantum Systems with Finite Hilbert Space. Reports on Progress in Physics, 67, 1. https://doi.org/10.1088/0034-4885/67/3/R03

[2] Cotfas, N. and Gazeau, J.P. (2010) Quantum Systems with Finite Hilbert Space and Frame Quantization. Journal of Physics A, 43, Article ID: 193001.

[3] Durt, T., Englert, B.G., Bengtsson, I. and Zyczkowski, K. (2010) On Mutually Unbiased Bases. International Journal of Quantum Information, 8, 535-640. https://doi.org/10.1142/S0219749910006502

[4] Tolar, J. and Chadzitaskos, G. (2009) Feynman's Path Integral and Mutually Unbiased Bases. Journal of Physics A, 42, Article ID: 245306. https://doi.org/10.1088/1751-8113/42/24/245306

[5] Gibbons, K., Hoffman, M.J. and Wootters, W. (2004) Discrete Phase Space Based on Finite Fields. Physical Review A, 70, Article ID: 062101. https://doi.org/10.1103/PhysRevA.70.062101

[6] Klappenecker, A. and Rotteler, M. (2004) Constructions of Mutually Unbiased Bases. Lecture Notes in Computer Science, 2948, 137-144. https://doi.org/10.1007/978-3-540-24633-6_10

[7] Saniga, M. and Planat, M. (2006) Hjelmslev Geometry of Mutually Unbiased Bases. Journal of Physics A, 39, 435. https://doi.org/10.1088/0305-4470/39/2/013

[8] Sulc, P. and Tolar, J. (2007) Group Construction of Mutually Unbiased Bases. Journal of Physics A: Mathematical and Theoretical, Article ID: 15099.

[9] Albouy, O. (2009) The Isotropic Lines of $\mathcal{Z}_{d}^{2}$. Journal of Physics A: Mathematical and Theoretical, 42, Article ID: 072001. https://doi.org/10.1088/1751-8113/42/7/072001

[10] Shalaby, M. and Vourdas, A. (2012) Tomographically Complete Set of Orthonormal Bases. Journal of Physics A, 45, Article ID: 052001.

[11] Shalaby, M. and Vourdas, A. (2013) Mutually Unbiased Projectors and Duality between Lines and Bases in Finite Quantum Systems. Annals of Physics, 337, 208-220. 
https://doi.org/10.1016/j.aop.2013.06.018

[12] Oladejo, S.O., Lei, C. and Vourdas, A. (2014) Partial Ordering of Weak Mutually Unbiased Bases. Journal of Physics A: Mathematical and Theoretical, 47, Article ID: 485204. https://doi.org/10.1088/1751-8113/47/48/485204

[13] Batten, L.M. (1997) Combinatorics of Finite Geometries. Cambridge University Press, Cambridge.

[14] Hirchfeld, J.W.P. (1979) Projective Geometries over Finite Fields. Oxford University Press, Oxford.

[15] Planat, M., Saniga, M. and Kibler, M.R. (2006) SIGMA, 2, 66.

[16] Havlicek, H. and Saniga, M. (2008) Projective Ring Line on a Specic Qudits. Journal of Physics A, 41, Article ID: 015302.

[17] Good, I.J. (1971) The Relationship between Two Fast Fourier Transforms. IEEE Transactions on Computers, C-20, 310. https://doi.org/10.1109/T-C.1971.223236

[18] Vourdas, A. and Banderier, C. (2010) Symplectic Transformations and Quantum Tomography in Finite Quantum Systems. Journal of Physics A, 43, Article ID: 042002.

[19] Durt, T. (2005) About Mutually Unbiased Bases in Even and Odd Prime Power Dimensions. Journal of Physics A: Mathematical and General, 38, 5267. https://doi.org/10.1088/0305-4470/38/23/013

[20] Zak, J. (2011) Doubling Feature of Wigner Function Finite Space. Journal of Physics $A$, 44, Article ID: 345305.

[21] Zak, J. (2012) Inversion Operators in Finite Phase Plane. Journal of Mathematical Physics, 53, Article ID: 103514. https://doi.org/10.1063/1.4752731 